\title{
Stationary ideal flow on a free surface of a given shape
}

\section{Tophøj, Laust Emil Hjerrild; Bohr, Tomas}

Published in:

Journal of Fluid Mechanics

Publication date:

2013

Document Version

Publisher's PDF, also known as Version of record

Link back to DTU Orbit

Citation (APA):

Tophøj, L. E. H., \& Bohr, T. (2013). Stationary ideal flow on a free surface of a given shape. Journal of Fluid Mechanics, 721, 28-45.

\section{General rights}

Copyright and moral rights for the publications made accessible in the public portal are retained by the authors and/or other copyright owners and it is a condition of accessing publications that users recognise and abide by the legal requirements associated with these rights.

- Users may download and print one copy of any publication from the public portal for the purpose of private study or research.

- You may not further distribute the material or use it for any profit-making activity or commercial gain

- You may freely distribute the URL identifying the publication in the public portal

If you believe that this document breaches copyright please contact us providing details, and we will remove access to the work immediately and investigate your claim. 


\title{
Stationary ideal flow on a free surface of a given shape
}

\author{
L. Tophøj and T. Bohr $\dagger$ \\ Physics Department \& Center for Fluid Dynamics, The Technical University of Denmark, \\ Kgs. Lyngby, DK-2800, Denmark
}

(Received 14 March 2012; revised 23 October 2012; accepted 15 January 2013; first published online 13 March 2013)

\begin{abstract}
We study the stationary, ideal flow on a free fluid surface with a prescribed shape. It is demonstrated that the flow is governed by a self-contained set of equations for the surface flow field without any reference to the bulk flow. To write down these equations for arbitrary surfaces, we apply a covariant formulation using Riemannian geometry and we show how to include surface tension and velocity-dependent forces such as the Coriolis force. We write down explicitly the equations for cases where the surface elevation can be written as function of either Cartesian or polar coordinates in the plane, and we obtain solutions for the important case of rotational symmetry and the perturbed flow when this symmetry is slightly broken. To understand the general character and solubility of the equations, we introduce the associated dynamical system describing the motion along the streamlines. The existence of orbits with transversal intersections, as well as quasi-periodic and chaotic solutions, show that not all boundary value problems are well-posed. In the particular case of unforced motion the streamlines are geodesic curves and in this case the existence of a nontrivial surface velocity field requires that the surface can be foliated by a family of non-intersecting geodesic curves.
\end{abstract}

Key words: Hamiltonian theory, mathematical foundations, rotating flows

\section{Introduction}

The close connection between surface flow and surface deformation is well known from everyday experiences as well as from laboratory experiments in fluid dynamics. For example, the localized surface deformation behind an oar is closely linked to the swirling motion on the surface of the vortices in the wake. One might then ask how strong this link is: Is it possible from knowledge of the shape of the surface to infer the surface flow? In this paper we shall show that the answer is to a large extent affirmative.

In $\S 2$ we present a simple observation, which to our knowledge has not been stated clearly before: that the stationary flow of an ideal incompressible fluid on a stationary free surface is governed by self-contained dynamical equations that do not involve the flow outside the free surface. These equations are the projection of the Euler equation onto the local surface tangent plane. Note that this does not imply that we can necessarily solve a given hydrodynamical problem by this method. The fact that 
the surface is 'free', means that it is free to move to the position where the appropriate boundary conditions are satisfied, and to compute this position, i.e. the height profile, we have to solve the equations in the entire bulk domain. However, once this surface shape is known (e.g. from observations) one can compute the flow on the surface without worrying about the bulk flow.

To write these equations in a transparent way and connect them to the geometry of the surface, we use a covariant description where the free surface is described as a two-dimensional Riemannian manifold equipped with a metric tensor. With this formalism, which is reviewed in $\S 3$, one can readily write the surface flow equations on an arbitrary smooth free surface. This is done in $\S 4$. The equations can be made to account for surface tension and velocity-dependent forces, such as the Coriolis acceleration in a non-inertial reference frame.

In $\S 5$, we move on to describe a situation where the flow and surface shape possess invariance under rotations about a fixed axis, a case that has been discussed in Bergmann et al. (2011). In this case the equations simplify a great deal and it is straightforward to find solutions. The types of solutions demonstrate a crucial property of the surface flow equations: that they may be singular on lines separating regions with different behaviour of the flow. The ramifications for general surface flows with rotational invariance is discussed. We next study flows in which the circular symmetry is broken, and as an example we look at the perturbation expansion for a line vortex on a slightly asymmetric surface. We obtain expressions for the streamlines and discuss the conditions for the absence of drift.

In $\S 6$ we show that the surface flow equations can be interpreted as a dynamical system, where particles of the dynamical system move along the characteristics for the field equations. With no external forcing (like gravity) the orbits are geodesics corresponding to the given surface deformation. We give general expressions for the Lagrangian and the Hamiltonian controlling the dynamics, and we relate the Bernoulli integral to the conserved energy along the orbit.

Finally, in $\S 7$ we discuss the general solubility of the surface flow equations. In the example treated in $\S 5$, we gave explicit formulae for flow velocities and streamlines for a slightly perturbed line vortex. On the other hand, the dynamical orbits introduced in $\S 6$, will, since they are projections from the energy surface of a four-dimensional phase space, generally intersect, and the existence of a well-defined velocity field on the surface thus depends crucially on the structure of the domain and the boundary conditions imposed on it. As an example, we treat an anisotropic quadratic surface elevation and show that the perturbation expansion breaks down.

\section{Decoupling of the free-surface Euler equations from the bulk flow}

Consider the stationary flow of an inviscid, incompressible fluid of constant density $\rho_{0}$. The velocity vector is denoted by $\boldsymbol{v}$ and the vorticity vector is $\boldsymbol{\omega}=\boldsymbol{\nabla} \times \boldsymbol{v}$. Conservation of momentum for time-independent flow is expressed by the Euler equation,

$$
(\boldsymbol{v} \cdot \nabla) \boldsymbol{v}=-\frac{1}{\rho_{0}} \nabla p+\boldsymbol{f} .
$$

External body forces are represented by the acceleration vector $f$. Note that $p$ is the pressure divided by the constant fluid density.

On a smooth free surface, inviscid flow governed by (2.1) is subject to: (a) the kinematic boundary condition, that $\boldsymbol{v} \cdot \boldsymbol{n}=0$, where $\boldsymbol{n}$ is any normal vector to the 
surface; and (b) the dynamic boundary condition, that the pressure (neglecting an immaterial constant term) is $p=2 \gamma H$. This is the Young-Laplace pressure associated with constant surface tension $\gamma$ on a surface of mean curvature $H$, which is welldefined at every point on the free surface and differentiable in terms of any smooth surface parametrization.

We use the identity $(\boldsymbol{v} \cdot \nabla) \boldsymbol{v}=\boldsymbol{\nabla}\|\boldsymbol{v}\|^{2} / 2+\boldsymbol{\omega} \times \boldsymbol{v}$ and project (2.1) along a tangent vector $\boldsymbol{t}$ to the surface by taking the dot product of $\boldsymbol{t}$ and the vector quantities on either side. The resulting equation, valid in the surface, is

$$
(\boldsymbol{t} \cdot \nabla) \frac{\|\boldsymbol{v}\|^{2}}{2}+\boldsymbol{t} \cdot(\boldsymbol{\omega} \times \boldsymbol{v})=-2 \frac{\gamma}{\rho_{0}}(\boldsymbol{t} \cdot \nabla) H+\boldsymbol{t} \cdot \boldsymbol{f} .
$$

Aside from the triple product term $\boldsymbol{t} \cdot(\boldsymbol{\omega} \times \boldsymbol{v})$, all the terms in (2.2) are explicitly in the tangent plane since $\boldsymbol{t} \cdot \boldsymbol{\nabla}$ is the derivative along a tangent vector in the surface, and since the kinematic boundary condition ensures that the normal component of $v$ vanishes in the surface. To show that the triple product is also in the tangent plane, we use the invariance of a triple product under cyclic permutations of the factors: $\boldsymbol{t} \cdot(\boldsymbol{\omega} \times \boldsymbol{v})=\omega \cdot(\boldsymbol{v} \times \boldsymbol{t})$. Now, $\boldsymbol{v} \times \boldsymbol{t}$ (a cross-product of tangent vectors) is clearly normal to the surface, so (2.2) refers only to the normal component of the vorticity field, which in turn is defined by velocity gradients only in the tangent plane.

We have then achieved a decoupling between the surface flow and the bulk flow in the sense that (2.2) involves only velocity components and derivatives in the surface. Note that the continuity equation, which involves the normal derivative of the normal component of $\boldsymbol{v}$ is left indeterminate, but since the pressure has disappeared as a variable, the surface flow equations, being basically two coupled equations for two independent surface velocity components, are sufficient for a solution. This will be discussed in greater detail later. Note at this point, that knowledge of the surface flow allows the determination of the normal derivative $\partial v_{n} / \partial n$ of the normal velocity by the continuity equation,

$$
\boldsymbol{\nabla} \cdot \boldsymbol{v}=\frac{\partial v_{n}}{\partial n}+\nabla_{s} \cdot \boldsymbol{v}=0,
$$

where $\nabla_{s} \cdot v$ is the divergence in the tangent plane.

Let us briefly discuss the case of a viscous flow. The dynamical boundary condition requires the viscous stress tensor to vanish on the free surface. However, the divergence of the stress tensor, which gives the viscous force density, need not vanish. The uncoupling cannot take place for the viscous Navier-Stokes equation as one can immediately realize by considering the Poiseuille flow of a uniform film of liquid flowing steadily down an inclined plate. At the free surface, the force of gravity is balanced by the viscous force, a necessary condition for steady flow. In many cases, however, it is useful to approximate the dynamics of a given flow by neglecting viscous stresses. This would require that the Reynolds number be small, i.e. that the viscous force term is small compared to the terms included in (2.1). Even though the flow of a real flow is often dramatically different from a solution to the Euler equation, this difference is typically localized to strong vorticity in boundary layers near solid boundaries, and regions where such boundary layer vorticity is advected by the flow. For extended regions of the fluid, viscosity plays a negligible role in the local dynamics.

Note that we could easily have considered a time-dependent velocity field. The inclusion of the term $\partial \boldsymbol{v} / \partial t$ in (2.2) would still allow us to proceed with the projection to the tangent plane. We have not pursued this further here since in applications this 
would typically imply that the surface shape is time dependent too, and this would introduce severe complications.

\section{Covariant formulation of the three-dimensional Euler equations}

In the previous section we showed that the Euler equation, when projected along the free surface, decouples from the bulk flow. This was done locally, with reference to vectors defined in the tangent plane associated with a specific point on the free surface. Now, in order to assemble this point-wise information into a useful set of partial differential equations we shall proceed to develop a framework based on tensor notation and the description of the free surface as a two-dimensional Riemannian manifold. In many cases, the dynamical equations can be derived and analysed without reference to the physical three-dimensional space, into which this manifold is embedded. However, inclusion of magnetic or Coriolis forces, and the effect of surface tension, require us to refer to the details of the embedding.

Conventionally, the equations of fluid mechanics are written in terms of orthogonal coordinates. When dealing with the flow on a (generally curved) surface, it is generally practical to use non-orthogonal coordinates. Even though orthogonal coordinate systems do exist for any two-dimensional smooth manifold, cf. Stoker (1969), their relation to typical laboratory coordinates can be complicated, and their very definition may involve the solution of differential equations. Riemannian geometry, on the other hand, provides a straightforward procedure for describing the flow on any smooth surface using non-orthogonal coordinates. For example, a wavy surface given in Cartesian coordinates $(x, y, h(x, y))$, where $h$ is the surface height, can immediately be described as a manifold parameterized by the coordinates $(x, y)$, which are nonorthogonal when the surface has a height gradient at an angle to the coordinate axes. In the following, we shall develop a procedure for writing the projected Euler equations governing free-surface flows on such (and more general) surfaces. For a physicist's introduction to Riemannian geometry, see Carroll (2003) or, specifically applied to fluid mechanics, see Aris (1962). We start by writing the full threedimensional stationary Euler equation in covariant form. Covariance means that the equations keep their meaning when the coordinate system is transformed. The essential point is that we write our equations in terms of tensors, defined as objects that transform according to specific rules, implying covariance. If the equations hold in one coordinate system, they will hold in another, due to the tensor property. So we need simply write the Euler equation in tensor form, such that it reduces to the well-known expression in orthogonal coordinates.

First we introduce an orthogonal 'laboratory' coordinate system $y^{\alpha}=\left(y^{1}, y^{2}, y^{3}\right)$. The corresponding metric tensor is $\delta_{\alpha \beta}$, the Kronecker delta. Let the fluid domain be described by a set of coordinates $x^{\mu}, \mu=1,2,3$, given a transformation

$$
x^{\mu} \rightarrow y^{\alpha}\left(x^{\mu}\right)
$$

and a metric tensor

$$
g_{\mu \nu}=\frac{\partial y^{\alpha}}{\partial x^{\mu}} \frac{\partial y^{\beta}}{\partial x^{\nu}} \delta_{\alpha \beta} .
$$

By convention, summation over repeated indices is implied. In the following, we shall use Greek letters to denote indices over three-dimensional space and Latin letters to denote indices over the two-dimensional manifold describing the free surface. The inverse metric $g^{\mu \nu}$ is defined as the matrix inverse of $g_{\mu \nu}$, i.e. $g_{\mu \lambda} g^{\lambda \nu}=\delta_{\mu}^{\nu}$. 
A vector is a quantity with one upper index, e.g. the fluid flow velocity $V^{\mu}=\mathrm{d} x^{\mu} / \mathrm{d} t$, where $x^{\mu}$ are the coordinates of a fluid particle. A dual vector is a quantity with a lower index, e.g. the gradient $\partial_{\nu} \Phi \equiv \partial \Phi / \partial x^{\nu}$ of a scalar $\Phi$. Both are tensors and transform accordingly, cf. Carroll (2003). The rules of tensor manipulation ensure that contractions, e.g. $a_{\mu} \equiv g_{\mu \nu} V^{\nu}$, or products, e.g. $b^{\mu}{ }_{\nu} \equiv V^{\mu} \partial_{\nu} \Phi$, are tensors. We can raise and lower indices by the metric tensor, i.e. we can define a dual velocity vector by $V_{\mu} \equiv g_{\mu \nu} V^{\nu}$ or a vector gradient by $\partial^{\mu} \Phi \equiv g^{\mu \nu} \partial_{\nu} \Phi$. The physical velocity vector $\boldsymbol{v}$ is given in terms of $V^{\mu}$ by $\boldsymbol{v}=\boldsymbol{e}_{\mu} V^{\mu}$, where $\boldsymbol{e}_{\mu}=\partial \boldsymbol{y} / \partial x^{\mu}$ are the dual basis vectors. Note that $\boldsymbol{e}_{\mu}$ need not be either unit vectors nor mutually orthogonal.

The Euler equation in covariant form reads

$$
V^{\nu} \nabla_{\nu} V^{\mu}=-g^{\mu \nu} \frac{\partial \Phi}{\partial x^{\nu}}+f^{\mu}
$$

The left-hand side of (3.3) is the covariant expression of the conventional advective derivative and contains the covariant derivative

$$
\nabla_{\nu} V^{\mu}=\frac{\partial V^{\mu}}{\partial x^{\nu}}+\Gamma_{\nu \lambda}^{\mu} V^{\lambda}
$$

The last term of (3.4) is a curvature term that accounts for the change of coordinate directions over space. They contain the Christoffel connection coefficients, defined in terms of the metric by

$$
\Gamma_{\mu \nu}^{\lambda}\left[g_{\alpha \beta}\right]=\frac{1}{2} g^{\lambda \sigma}\left(\frac{\partial g_{v \sigma}}{\partial x^{\mu}}+\frac{\partial g_{\mu \sigma}}{\partial x^{\nu}}-\frac{\partial g_{\mu \nu}}{\partial x^{\sigma}}\right),
$$

where we have explicitly written the dependence on the metric $g_{\alpha \beta}$. The connection coefficients are not tensors, but the covariant derivative defined by (3.4) is. On the right-hand side of (3.3) we have the scalar potential field $\Phi$ and the applied force $f^{\mu}$. In $\Phi$ we can e.g. include gravity forces (a linear term in $h$ in a constant gravitational field), centrifugal forces, and surface tension (a term proportional to the surface mean curvature). The covariant formulation of the Euler and Navier-Stokes equations has been discussed earlier, e.g. by Ilin (1991).

To see that (3.3) are indeed the stationary Euler equations, we inspect their form in Cartesian coordinates, where $g_{\mu \nu}=\delta_{\mu \nu}$. Then $\Gamma_{\mu \nu}^{\lambda}=0$, and the equations read $(\boldsymbol{v} \cdot \nabla) \boldsymbol{v}=-\nabla \Phi+f$, which we recognize.

\section{Covariant formulation of the surface flow equations}

In order to project (3.3) onto the free surface, we shall now introduce a particular set of surface-adapted coordinates. We denote the free surface by $\mathscr{S}$. We assume that $\mathscr{S}$ is described by a smooth regular parameterized surface, i.e. a map $f_{0}: x^{i} \rightarrow y^{\alpha}$

$$
x^{i} \rightarrow y^{\alpha} \equiv f_{0}^{\alpha}\left(x^{i}\right) \text {. }
$$

Here and below we use Greek letters to denote indices over three-dimensional space, so $x^{\mu}=\left(x^{1}, x^{2}, x^{3}\right)$, and Latin letters for indices over $\mathscr{S}$, so $x^{i}=\left(x^{1}, x^{2}\right)$. The regularity of the parameterization (4.1) means that at any point, the vectors $\partial f_{0}^{\alpha} / \partial x^{1}$ and $\partial f_{0}^{\alpha} / \partial x^{2}$ are linearly independent, and so they span the local tangent plane. The smoothness condition means that the tangent plane varies smoothly with $\left(x^{1}, x^{2}\right)$. As sketched in figure 1 , we introduce the dual vectors, defined on $\mathscr{S}$,

$$
\boldsymbol{e}_{1}\left(x^{i}\right)=\frac{\partial f_{0}^{\alpha}}{\partial x^{1}}, \quad \boldsymbol{e}_{2}\left(x^{i}\right)=\frac{\partial f_{0}^{\alpha}}{\partial x^{2}}, \quad \boldsymbol{e}_{3}\left(x^{i}\right)=\frac{\boldsymbol{e}_{1} \times \boldsymbol{e}_{2}}{\left\|\boldsymbol{e}_{1} \times \boldsymbol{e}_{2}\right\|} .
$$



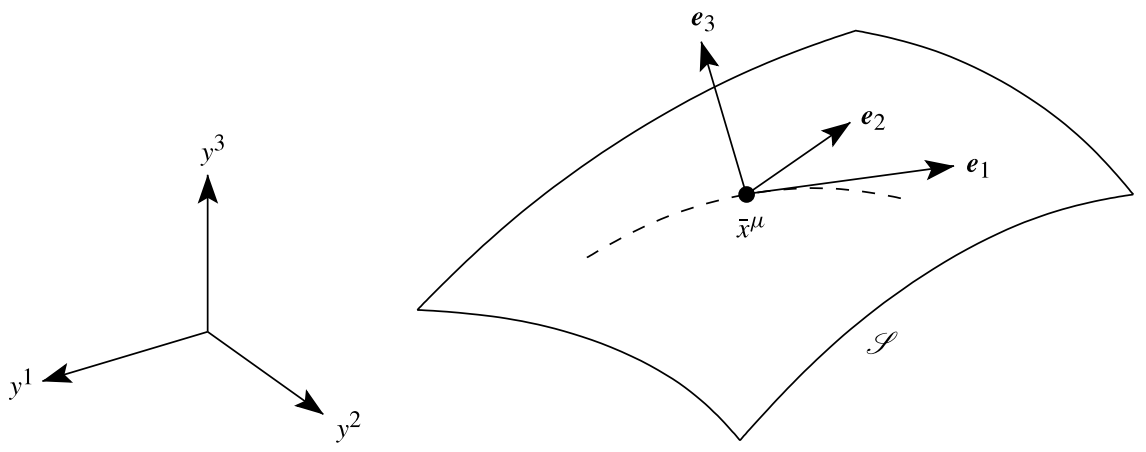

FIGURE 1. Sketch of the coordinates: $y^{\alpha}$ are the Cartesian laboratory coordinates; $x^{\mu}$ are nonorthogonal coordinates attached to the free surface $\mathscr{S}$ and defined by the map $y^{\alpha}=f^{\alpha}\left(x^{\mu}\right)$ given in (4.3). The basis dual vectors $\boldsymbol{e}_{i},(4.2)$, associated with a point $x^{\mu}=\bar{x}^{\mu}$ define the local coordinate directions, in the sense that $f^{\alpha}\left(\bar{x}^{\mu}+\mathrm{d} x^{\mu}\right)=f^{\alpha}\left(\bar{x}_{0}\right)+\mathrm{d} x^{\mu} \boldsymbol{e}_{\mu}\left(x_{0}^{\mu}\right)$. The dashed line tangent to $\boldsymbol{e}_{1}$ is obtained by varying $x^{1}$ while keeping $x^{2}$ and $x^{3}$ fixed. Equation (4.3) defines a valid coordinate system close to $\mathscr{S}$, i.e. for small values of $x^{3}$.

Then the map $f: x^{\mu} \rightarrow y^{\alpha}$,

$$
f^{\alpha}\left(x^{1}, x^{2}, x^{3}\right)=f_{0}^{\alpha}\left(x^{i}\right)+x^{3} \boldsymbol{e}_{3}\left(x^{i}\right),
$$

defines a coordinate system on and near the surface $\mathscr{S}$. It defines a coordinate system for small values of $\left|x^{3}\right|$ because $f$ is a regular invertible map between a neighbourhood in $\mathbb{R}^{3}$ of any point $\left(x^{1}, x^{2}, 0\right)$ and a neighbourhood in physical space of the point $f_{0}^{\alpha}\left(x^{1}, x^{2}\right)$. This can be seen by computing the Jacobian matrix of $f$ and using the inverse function theorem and this argument extends to the case where $\mathscr{S}$ is described by overlapping charts. We find the corresponding metric tensor from (3.2),

$$
g_{\mu \nu}=\boldsymbol{e}_{\mu} \cdot \boldsymbol{e}_{\nu}+x^{3}\left(\boldsymbol{e}_{\mu} \cdot \frac{\partial \boldsymbol{e}_{3}}{\partial x^{\nu}}+\boldsymbol{e}_{\nu} \cdot \frac{\partial \boldsymbol{e}_{3}}{\partial x^{\mu}}\right) .
$$

Now, (4.4) allows us to compute the first derivatives of the metric on $\mathscr{S}$ and so the Christoffel symbols in (3.5). Due to the property $\boldsymbol{e}_{i} \cdot \boldsymbol{e}_{3}=0$, (4.4) takes a special form on $\mathscr{S}$, essentially decomposing the part pertaining to $x^{1}, x^{2}$ :

$$
g_{\mu \nu}=\left(\begin{array}{ccc}
{\left[g_{i j}\right]} & 0 \\
0 & 0 & 1
\end{array}\right),
$$

where $g_{i j}$ is the two-dimensional metric tensor of the surface submanifold:

$$
g_{i j}=\frac{\partial f_{0}^{\alpha}}{\partial x^{i}} \frac{\partial f_{0}^{\beta}}{\partial x^{j}} \delta_{\alpha \beta}, \quad i, j=1,2 .
$$

Similarly, we introduce submanifold connection coefficients $\Gamma_{j k}^{i}\left[g_{l m}\right]$ by restricting the summation indices in (3.5) to 1,2 . We note that these new connection coefficients are identical to the corresponding ones defined from the full metric $\Gamma_{j k}^{i}\left[g_{l m}\right]=\Gamma_{j k}^{i}\left[g_{\mu \nu}\right]$, but we want to stress that they can be computed without reference to the $x^{3}$ coordinate. The block form of $g_{\mu \nu}$ in (4.5) implies that the inverse metric $g^{\mu \nu}$ takes a similar block form, with $g_{i j}$ in (4.5) replaced by its inverse, $g^{i j}$. 
We shall now show that, in accordance with $\S 2$, the equations of (3.3) for $\mu=1,2$, refer only to the physical fields directly on the free surface, and furthermore, that one can determine the coordinate form of these equations without bothering to even define the third coordinate, $x^{3}$. We assume for now that the force and potential fields $f^{i}$ and $\Phi$ are known on the surface as functions of the parameterizing coordinates $x^{i}$.

The kinematic boundary condition, $\boldsymbol{v} \cdot \boldsymbol{n}=0$, means that the physical velocity vector $\boldsymbol{v}$ lies in the plane spanned by $\boldsymbol{e}_{1}, \boldsymbol{e}_{2}$. Since the linearly independent dual basis vectors $\boldsymbol{e}_{\mu}$ admit a unique linear combination forming $\boldsymbol{v}$, this implies $V^{3}=0$.

Looking at the terms of (3.3) with $\mu=1,2$, we note that the partial derivatives $\partial / \partial x^{i}$ refer only to surface quantities. Equation (4.5) implies $g^{i v} \partial \Phi / \partial x^{v}=g^{i j} \partial \Phi / \partial x^{j}$ so the potential term depends only on the potential defined on the free surface. As for the convective derivative $V^{v} \nabla_{v} V^{i}=V^{j} \nabla_{j} V^{i}$, it contains only surface-directed velocity derivatives $\partial V^{i} / \partial x^{j}$ and the connection coefficients $\Gamma_{j k}^{i}$, which may be computed directly from the surface metric (4.6).

Let us return to the question of the potential and force fields, which must be defined using the full map $x^{i} \rightarrow y^{\alpha}$. A crucial feature of the surface equations is, in contrast to the three-dimensional Euler equations (3.3), that the pressure drops out of the potential, since it is constant on the surface, except for the Laplace pressure due to surface tension. We thus assume that the surface potential has the form

$$
\Phi=\phi\left(y^{\alpha}\right)+2 \gamma H
$$

where $\phi$ could include gravity, centrifugal forces and electrostatic forces and where $H$ is the surface mean curvature defined on the surface. $H$ is positive when the surface curves in the direction of the normal vector $\boldsymbol{e}_{3}$. Physically, this corresponds to $\boldsymbol{e}_{3}$ pointing out of the fluid. For example $y^{\alpha}=\left(x^{1}, x^{2}, h\left(x^{1}, x^{2}\right)\right)$ corresponds to the typical situation where the fluid is below the interface.

The mean curvature is given by

$$
H\left(x^{i}\right)=-\frac{1}{2} g^{\mu \nu} \nabla_{\nu} n_{\mu}=-\frac{1}{4} g^{\mu \nu} \frac{\partial g_{\mu \nu}}{\partial x^{3}},
$$

where $n_{\mu}=\delta_{\mu}^{3}$ is the dual normal vector field. In (4.8), we have used the extrinsic curvature tensor $\nabla_{\mu} n_{\nu}$ associated with the surface, a generalization of the second fundamental form of the surface, cf. Carroll (2003). The tensor $g^{\mu \lambda} \nabla_{\lambda} n_{v}$ generalizes the Weingarten matrix, the trace of which is associated with the mean curvature, cf. do Carmo (1976). Note that $H$ is an extrinsic quantity, i.e. it depends on the metric outside the free surface by the tensor $\partial g_{\mu \nu} / \partial x^{3}$ given in (4.4). The definitions in (4.1)-(4.2) and (4.5) allow $H$ to be computed as a function of $x^{i}$.

Now, using (4.1) again, we can define the potential (4.7) as a function of the surface coordinates by

$$
\Phi\left(x^{i}\right)=\phi\left(y^{\alpha}\left(x^{i}\right)\right)+2 \gamma H\left(x^{i}\right) .
$$

Similarly, we compute the Coriolis acceleration term, $f^{\mu}$ in (3.3). The cross-product $\boldsymbol{A}=\boldsymbol{B} \times \boldsymbol{C}$ in terms of covariant coordinates is

$$
A^{\mu}=g^{\mu \nu} \sqrt{g} \varepsilon_{v \rho \sigma} B^{\rho} C^{\sigma},
$$

where $\sqrt{g}=\left|g_{\mu \nu}\right|^{1 / 2}$ is the determinant of the Jacobian matrix $\partial y^{\alpha} / \partial x^{\mu}$, and $\varepsilon_{v \rho \sigma}$ is the Levi-Civita symbol, equal to the sign of the permutation of the numbers $v \rho \sigma$ and zero if any number is repeated. Equation (4.10) can be derived using elementary techniques by considering a linear invertible map to Cartesian coordinates and comparing to the well-known formula. For a more conventional differential geometry derivation in terms 
of dyadics, cf. Lebedev, Cloud \& Eremeyev (2010). Note that $A^{\mu}$ is a pseudovector, i.e. it switches sign in a left-handed coordinate system. Using (4.5) and the kinematic boundary condition, we see that the components $f^{i}$ of (4.10) applied to $\boldsymbol{f}=-2 \boldsymbol{\Omega} \times \boldsymbol{v}$ refer only to the third component $\Omega^{3}=\boldsymbol{e}_{3} \cdot \Omega$ of $\Omega$, and we have

$$
f^{i}\left(x^{k}\right)=2\left|g_{i j}\right|^{1 / 2} \Omega^{3}\left(g^{i 1} V^{2}-g^{i 2} V^{1}\right) .
$$

With all this in place, we are ready to define the flow equations in terms of the manifold coordinates $x^{i}$.

In summary, the ideal flow on a stationary free surface parametrized by $\left(x^{1}, x^{2}\right) \rightarrow$ $\left(y^{1}, y^{2}, y^{3}\right)$ is governed by the momentum balance equations

$$
V^{j} \nabla_{j} V^{i}=V^{j} \frac{\partial V^{i}}{\partial x^{j}}+\Gamma_{j k}^{i} V^{j} V^{k}=-g^{i j} \frac{\partial \Phi}{\partial x^{j}}+f^{i},
$$

where $i, j=1,2$, and the metric is defined by (4.6). The left-hand side can be computed from the metric alone, using (3.4) and $\Gamma_{j k}^{i}\left[g_{l m}\right]$ defined by (3.5). The terms on the right-hand side depend on the embedding $x^{i} \rightarrow y^{\alpha}$ and the dual basis (4.2). Expressions for the potential $\Phi\left(x^{i}\right)$ and the Coriolis acceleration $f^{i}\left(x^{j}\right)$ are given in (4.9) and (4.11). In the Appendix we give the coordinate expression for (4.12) in two important cases, where the horizontal position is described by the coordinates $\left(x^{1}, x^{2}\right)$, which are either Cartesian or polar, and the vertical height is given by $y^{3}=h\left(x^{1}, x^{2}\right)$.

We shall later see that (4.12) has a first integral generalizing the Bernoulli field, see (6.7a) below.

As for the possibility of specifying the normal derivative $\partial v_{n} / \partial n$ of the normal velocity by the continuity equation, cf. $\S 2$, we note that the covariant formulation of the continuity equation (2.3) is

$$
\nabla_{\mu} V^{\mu}=0 .
$$

On the free surface, where $V^{3}=0$, the only quantities referring to fields outside the surface (4.13) are $\partial V^{3} / \partial x^{3}$ and $\Gamma_{3 i}^{3}=0$, where the last equality follows from the coordinate definition (4.4). So we are left with the following expression for the normal derivative:

$$
\frac{\partial v_{n}}{\partial n}=\frac{\partial V^{3}}{\partial x^{3}}=-\frac{\partial V^{i}}{\partial x^{i}}-\Gamma_{i j}^{i} V^{j},
$$

where the last term is the curvature correction to (2.3).

\section{Solutions to the surface flow equations in symmetric and nearly symmetric situations}

We have seen how to write the surface flow equation (4.12) on a general curved surface with a general potential field, but to solve these equations, or even write them in coordinate form, is not an easy task. We shall therefore start by looking at a couple of simple special cases.

First we consider the special case when both the free-surface height and the flow field are invariant under rotations about the $y^{3}$-axis. In this case, (4.12) reduces to a simple set of two ordinary differential equations, and the solution is straightforward. The free surface is parameterized by the coordinates $\left(x^{1}, x^{2}\right)=(\rho, \theta)$. The corresponding (contravariant) velocity vector components are $\left(V^{1}, V^{2}\right) \equiv(U, V)$. Note that the vector component $V$ has the dimensions of an angular velocity. The 
position in space is given in terms of Cartesian coordinates $y^{\alpha}$ by

$$
\left(y^{1}, y^{2}, y^{3}\right)=(\rho \cos \theta, \rho \sin \theta, h(\rho)) .
$$

The potential field is $\Phi=\Phi(\rho)$. We do not include the possibility of a rotating reference frame at this stage, since any steady rotation about the symmetry axis may be included in the flow field. In the simplest case the potential includes only gravity and $\Phi(\rho)=\phi(\rho)=\rho_{0} g h(\rho)$, but the analysis below remains valid in the general case. Now, (4.12) written for polar base coordinates, see (A 10), yields

$$
\begin{aligned}
U U_{\rho}+\frac{1}{1+h_{\rho}^{2}}\left(h_{\rho} h_{\rho \rho} U^{2}-\rho V^{2}\right) & =-\frac{1}{1+h_{\rho}^{2}} \Phi_{\rho}, \\
U V_{\rho}+2 \frac{1}{\rho} U V & =0,
\end{aligned}
$$

for the $\rho$ - and $\theta$-directions, respectively. We use a subscript to denote differentiation, i.e. $U_{\rho}=\mathrm{d} U / \mathrm{d} \rho$. We can make sense of (5.2) by introducing the 'physical' velocity components $u, v$, along the dual basis $\boldsymbol{e}_{\rho}, \boldsymbol{e}_{\theta}$, so that the velocity vector is $\boldsymbol{v}=u \boldsymbol{e}_{\rho} /\left\|\boldsymbol{e}_{\rho}\right\|+v \boldsymbol{e}_{\theta} /\left\|\boldsymbol{e}_{\theta}\right\|$. In the symmetric situation, the coordinates are orthogonal, $\boldsymbol{e}_{\rho} \cdot \boldsymbol{e}_{\theta}=0$, so we have

$$
\begin{aligned}
& u=\left\|\boldsymbol{e}_{\rho}\right\| U=\sqrt{g_{\rho \rho}} U=\sqrt{1+h_{\rho}^{2}} U, \\
& v=\left\|\boldsymbol{e}_{\theta}\right\| V=\sqrt{g_{\theta \theta}} V=\rho V,
\end{aligned}
$$

in terms of which (5.2) are equivalent to

$$
\begin{aligned}
\frac{\mathrm{d}}{\mathrm{d} \rho}\left(\frac{u^{2}}{2}+\Phi\right)-\frac{v^{2}}{\rho} & =0, \\
\frac{u}{\rho^{2}} \frac{\mathrm{d}}{\mathrm{d} \rho}(\rho v) & =0 .
\end{aligned}
$$

An equivalent set of equations was derived in Bergmann et al. (2011) using a more pedestrian approach in line with our $\S 2$.

We will now briefly reiterate some analysis of Bergmann et al. (2011) and move on to some general considerations about the possible solutions to (5.4). First we note that (5.4b) is singular when $u=0$. Hence, the solutions to (5.4) fall into two categories:

(a) A regime where $u=0$, so $(5.4 b)$ is satisfied independently of $v$, and $(5.4 a)$ reduces to

$$
\frac{v^{2}}{\rho}=\Phi_{\rho}
$$

If the function $\Phi(\rho)$ is known, (5.5) directly expresses the possible forms $v(\rho)$. If on the other hand $v(\rho)$ is known, an expression for $\Phi(\rho)$ can easily be established by integrating (5.5).

(b) A regime where $u \neq 0$, and (5.4b) leads to

$$
v=\frac{\Gamma}{\rho},
$$


where $\Gamma$ is a constant, and the circulation along a streamline $\rho=$ constant is $2 \pi \Gamma$. Using (5.6a), (5.4a) integrates to

$$
\frac{1}{2}\left(u^{2}+v^{2}\right)+\Phi=\text { constant }
$$

which is the well-known Bernoulli equation connecting points along the same streamline in Euler flows, cf. (6.7a) below. This was to be expected, because in the symmetric case any finite region with $u \neq 0$ is connected by streamlines.

The splitting of the solutions to (5.4) presented above can be interpreted as follows. In regime $(a)$, different radii are not connected by streamlines, and $(5.4 b)$ is exhausted by $u=0$. That condition, however, allows us to directly solve $(5.4 a)$ either in terms of the velocity field or the potential field. In regime $(b)$, different radii are connected by streamlines, and (5.4) lead to conditions for the conservation of angular momentum $(5.6 a)$ and energy $(5.6 b)$.

Given a solution of either of the above types, we can compute the divergence of the surface flow from (4.14), which simplifies to

$$
\nabla_{j} V^{j}=-\frac{\partial v_{n}}{\partial n}=\frac{1}{\sqrt{1+h_{\rho}^{2}}}\left(\frac{u}{\rho}+\frac{\partial u}{\partial \rho}\right) .
$$

Solutions of type (a) are not associated with a surface divergence, but solutions of type (b) are, except in the case $\rho u=$ constant.

We now discuss the possible solutions to a flow problem, i.e. how to find functions $u(\rho)$ and $v(\rho)$ for a given function $\Phi(\rho)$. This can be tricky, as seen by the example in Bergmann et al. (2011), where physical arguments about the bulk flow have important implications for the surface flow.

For a given $h(\rho)$, and thus a given $\Phi(\rho)$, we can always find a solution of type (a) (with $u=0$ ) as long as $\Phi_{\rho} \geqslant 0$, simply by taking $v(\rho)=\sqrt{\rho \Phi_{\rho}}$ from (5.5). Furthermore, if $\Phi(\rho)$ is analytic in a domain including $\rho=0$, this is the only possible solution in the domain.

If for physical reasons we look for flows with a finite radial component, we must search for solutions of type $(b)$. This implies a line vortex flow, (5.6a). This solution cannot remain valid down to $\rho=0$, where it would blow up. One possibility is that there is a $\rho_{0}>0$, where the flow switches to a flow of type $(a)$ with $u=0$. If the flow should remain analytic at $\rho=0$, the potential at small $\rho$ should have an expansion of the form

$$
\Phi=\Phi_{0}+\frac{1}{2} A \rho^{2}+\cdots
$$

where we assume $A \neq 0$. In a constant gravitational field this would imply that the height has an extremum at $\rho=0$. Correspondingly, the velocity will be

$$
v=\frac{1}{2} A^{1 / 2} \rho+\cdots
$$

which shows that $A>0$ (i.e. the surface has a minimum) and that the flow approaches a rigid rotation near $\rho=0$.

Such behaviour is observed in Bergmann et al. (2011), where the flow is known to have a finite secondary (radial) component due to Ekman transport, a viscous effect in the bulk flow. In this case the flow changes from a line vortex flow for $\rho>\rho_{0}$ to a rigid rotation for $\rho<\rho_{0}$. On the singular locus, $\rho=\rho_{0}$, the velocity remains continuous, but the vorticity jumps discontinuously - at least in our somewhat idealized limit of zero viscosity. We speculate that such singular lines separating distinct flow types might not be uncommon in free surface flows. 
The results above can be used as a starting point for the investigation of flows where the free surface and the potential are nearly symmetric under rotations. Examples of such flows are bathtub vortices disturbed by waves travelling in the azimuthal direction, and the rotating polygon flows discussed by Bergmann et al. (2011). Proceeding from (A 10), one can introduce a nearly symmetric height profile $h \rightarrow H(\rho)+\varepsilon h(\rho, \theta)$, where $h(\rho, \theta)$ is a periodic function of $\theta$, and look for perturbed flow velocities $U(\rho, \theta)$ and $V(\rho, \theta)$ near the symmetric solutions (5.4). We shall not write down the perturbation expansion in this paper, but merely note that it is straightforward. As a new feature, one should, in general, expect drift in the solutions. Thus the streamlines satisfy

$$
\frac{\mathrm{d} \rho}{\mathrm{d} \theta}=\frac{U(\rho, \theta)}{V(\rho, \theta)}
$$

and if $\int_{0}^{2 \pi} U(\rho, \theta) / V(\rho, \theta) \mathrm{d} \theta \neq 0$, the streamline $\rho$ is not a periodic function of $\theta$, but contains a 'secular' term allowing a slow change in each revolution.

\section{The surface flow equations as a dynamical system}

We now discuss the possibility of solving the surface flow equation (4.12) and the difficulties that may arise. Unlike the ordinary Euler flow equation, (4.12) form a closed system, with no need for a continuity equation relating to either a condition of incompressibility or knowledge of the pressure field response to fluid compression, e.g. by an equation of state.

For a particle, whose position on the surface $x^{i}$ and velocity $V^{i}$ are functions of the time $t$, (4.12) can be viewed as a dynamical system, since by the chain rule

$$
\ddot{x}^{i} \equiv \frac{\mathrm{d} V^{i}}{\mathrm{~d} t}=\frac{\partial V^{i}}{\partial t}+V^{j} \frac{\partial V^{i}}{\partial x^{j}},
$$

and $V^{i}$ does not depend explicitly on time, i.e. $\partial V^{i} / \partial t=0$. So (4.12) implies that on the streamlines, the motion is governed by the two-degree-of-freedom dynamical system,

$$
\begin{aligned}
\dot{V}^{i} & =-\Gamma_{j k}^{i} V^{j} V^{k}-g^{i j} \frac{\partial \Phi}{\partial x^{j}}+f^{i}, \\
\dot{x}^{i} & =V^{i} .
\end{aligned}
$$

The dynamical system (6.2) is fundamentally different from the field equation (4.12). Given a solution to (4.12), i.e. a field $V^{i}\left(x^{j}\right)$, we could solve the two-dimensional ODE

$$
\dot{x}^{i}=V^{i}\left(x^{j}\right),
$$

where the unique velocity at a given point implies that there is a unique streamline passing through the point. On the contrary, (6.2) is a two-degree-of-freedom Hamiltonian system (a four-dimensional ODE), which has a much richer dynamics than (6.3), and it can for example have chaotic solutions. The trajectories $x^{i}=x^{i}(t)$ to (6.2) may intersect each other or even themselves, in violation of the condition that each point on the surface should have a unique velocity. So it is not straightforward to construct solutions to (4.12) from (6.2), and in some cases it is even impossible. We shall return to this discussion below, in $\S 7$.

Note that (6.2) corresponds to the Lagrangian

$$
L=\frac{1}{2} V_{j} V^{j}+V_{j} A^{j}-\Phi .
$$


The field $A^{j}=A^{j}\left(x^{i}\right)$ is analogous to the electromagnetic vector potential. Since the rotation vector is defined as a fixed direction in physical space, we must refer to the laboratory coordinates $y^{\alpha^{\prime}}$ defined just before (3.1). Here we indicate these coordinates by primed indices. In $y^{\alpha^{\prime}}$ coordinates, the vector potential is represented by the physical vector $\boldsymbol{A}=\boldsymbol{\Omega} \times \boldsymbol{y}$, whose components are $A^{\alpha^{\prime}} . A^{\alpha}$ is obtained through the vector transformation rule $A^{\alpha}=A^{\alpha^{\prime}} \partial x^{\alpha} / \partial y^{\alpha^{\prime}}$, where $\partial x^{\alpha} / \partial y^{\alpha^{\prime}}$ can be found by inverting $\partial y^{\alpha^{\prime}} / \partial x^{\alpha}$ given in (4.3). The Coriolis acceleration vector is related to $A_{j}$ by

$$
f^{i}=g^{i k} V^{j}\left(\frac{\partial A_{j}}{\partial x^{k}}-\frac{\partial A_{k}}{\partial x^{j}}\right) .
$$

The Lagrangian, (6.4), corresponds to the generalized momenta

$$
p_{i} \equiv \frac{\partial L}{\partial V^{i}}=V_{i}+A_{i},
$$

and the Hamiltonian

$$
\begin{aligned}
H & =p_{j} V^{j}-L \\
& =\frac{1}{2} V_{j} V^{j}+\Phi \\
& =\frac{1}{2} p_{j} p^{j}+\frac{1}{2} A_{j} A^{j}-p_{j} A^{j}+\Phi,
\end{aligned}
$$

where $p^{i} \equiv g^{i j} p_{i}$. Hamilton's equations of motion are then $\mathrm{d} x^{i} / \mathrm{d} t=\partial H / \partial p_{i}$ and $\mathrm{d} p_{i} / \mathrm{d} t=-\partial H / \partial x^{i}$.

Note that $(6.7 a)$ corresponds to the standard Bernoulli equation, which states that $H$ is constant along a streamline. This could also be seen from (4.12), which gives the directional derivative of $H$ along a streamline,

$$
V^{j} \frac{\partial H}{\partial x^{j}}=V^{j} \nabla_{j} H=V_{j} f^{j}=0,
$$

where the last equality follows from the Coriolis acceleration being orthogonal to $V^{i}$, cf. (4.11).

\subsection{The unforced case: geodesic flows}

Consider the nonlinear surface flow equation, (4.12), in the coordinate-independent form. We shall see that we can in some cases obtain knowledge of the general solution without even bothering to introduce a coordinate system. A special case occurs when $\Phi$ and $f$ vanish, and (6.2) simplifies to

$$
\frac{\mathrm{d}^{2} x^{i}}{\mathrm{~d} t^{2}}+\Gamma_{j k}^{i} \frac{\mathrm{d} x^{j}}{\mathrm{~d} t} \frac{\mathrm{d} x^{k}}{\mathrm{~d} t}=0 .
$$

This is the equation for a geodesic curve on the surface manifold, cf. Carroll (2003). So the solutions to (4.12) on a manifold comprise a family of non-intersecting geodesic curves.

\subsection{The nearly planar case}

Consider the case when the physical coordinates describing the free surface are $(x, y, h(x, y))$ for small heights $h$. Then the metric tensor for the surface manifold is given by (A 2), and

$$
g_{i j}=\delta_{i j}+O\left(h^{2}\right) .
$$


The correction term is of second order in $h$ and its gradient, and so are the connection coefficients $\Gamma_{j k}^{i}$. To lowest order in $h$ and expressed in terms of the physical velocity components $\boldsymbol{v}=(u, v)$, (4.12) becomes

$$
(\boldsymbol{v} \cdot \nabla) \boldsymbol{v}=-\boldsymbol{f} \times \boldsymbol{v}-\nabla \Phi,
$$

where the potential takes the form $\Phi=g_{0} h$ in a uniform gravity field of magnitude $g_{0}$. In traditional geophysical notation, we define the Coriolis parameter $\boldsymbol{f}$ as a vector pointing upwards out of the plane with $\|\boldsymbol{f}\|=2 \Omega \sin \phi$ on the geographical latitude $\phi$.

Now, (6.2) become

$$
\begin{aligned}
& \dot{\boldsymbol{v}}=-\boldsymbol{f} \times \boldsymbol{v}-\nabla \Phi, \\
& \dot{\boldsymbol{x}}=\boldsymbol{v} .
\end{aligned}
$$

These equations of motion correspond to the Lagrangian $L$ and Hamiltonian $H$,

$$
\begin{aligned}
L & =\frac{1}{2}\|\dot{\boldsymbol{x}}\|^{2}+\frac{1}{2} \dot{\boldsymbol{x}} \cdot(\boldsymbol{f} \times \boldsymbol{x})-\Phi, \\
H & =\frac{1}{2}\|\boldsymbol{p}\|^{2}+\frac{1}{8}\|\boldsymbol{f}\|^{2}\|\boldsymbol{x}\|^{2}-\frac{1}{2} \boldsymbol{p} \cdot(\boldsymbol{f} \times \boldsymbol{x})+\Phi,
\end{aligned}
$$

where the conjugate momenta are $p_{i} \equiv \partial L / \partial \dot{x}_{i}=\dot{x}_{i}+\varepsilon_{i 3 k} f x_{k}$. Hamilton's equations of motion are $\dot{p}_{i}=-\partial H / \partial x_{i}, \dot{x}_{i}=\partial H / \partial p_{i}$.

\section{Existence of solutions to the surface flow problem}

As we have seen, it is possible to solve the surface flow equations (4.12) for important special cases with symmetry and - at least perturbatively - when the symmetry is broken. As we shall now see, however, it is not guaranteed that a solution of (4.12) for a given surface shape and given boundary conditions exists at all. To analyse this question for various cases, we shall make frequent use of the dynamical systems description of the previous section.

As an example, where we can find solutions, consider a planar horizontal free surface with no vertical forcing. The particle orbits are geodesics, which according to (6.9) are straight lines. They must also be streamlines, so the only solution in a connected open subset of the plane is a parallel flow. The flow speeds on distinct streamlines are not related through (6.9), which contains only the acceleration of fluid particles along their path of motion, and obviously we can specify any parallel flow on (part of) the boundary.

Another example is a spherical drop of liquid. Without an external potential, and even in the presence of surface tension, the right-hand side of (4.12) vanishes, and we are again left with geodesic flows (6.9). The geodesics on the sphere are great circles. Any two non-identical great circles intersect each other at two points, and (4.12) has only one global solution, $V^{i}=0$. So the only equilibrium state for a spherical drop of inviscid liquid without external forcing is a state where the particles on the surface are at rest. Thus, in order to sustain a non-trivial flow the surface cannot remain totally flat, which shows the strong connection between surface shape and surface flow alluded to in the Introduction.

Ellipsoids can be analysed using a similar argument. A general triaxial ellipsoid has more complicated geodesics, cf. Arnold (1978, §47). A single geodesic is either a closed curve, or it is dense in an area between two confocal one-sheet hyperboloids. This behaviour is incompatible with the idea of a geodesic flow with $V^{i}=V^{i}\left(x^{j}\right)$. An analysis of surface conditions on equilibrium shapes of self-gravitating fluid bodies, e.g. Dedekind ellipsoids, could be pursued from (4.12) including a non-zero forcing. 
(a)

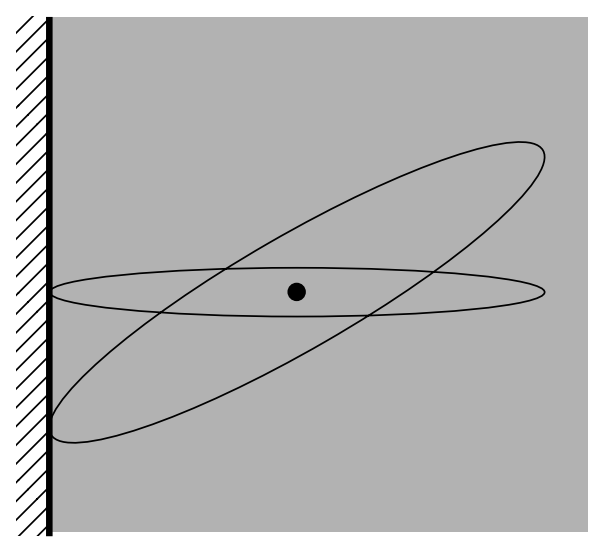

(b)

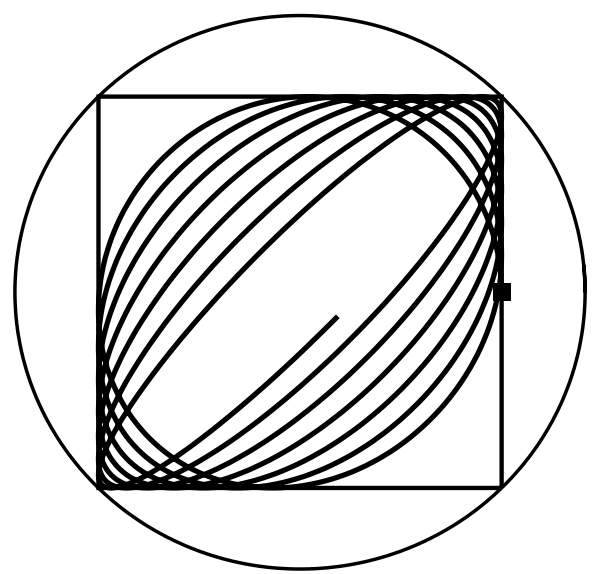

FIGURE 2. (a) Example of a boundary value problem in (6.11) with no solution. The parameters are given in (7.1). Any solutions to (6.12) trace ellipses centred at the origin (shown by the black dot). Impenetrable boundary conditions are applied at the line $x=-1$. A particle at a point $\boldsymbol{x}_{0}=(-1, y)$ at the boundary with velocity $\boldsymbol{v}=(0, v)$ will be advected according to (6.12) along an ellipse connecting the points $\boldsymbol{x}_{0}$ and $-\boldsymbol{x}_{0}$. Clearly, any two such trajectories emanating from distinct points on the boundary will intersect, and their velocities at the intersection points will not be identical. This is incompatible with the uniqueness of streamlines at each point of a stationary flow, and it seems that this boundary value problem has no solution at all. $(b)$ Position view $(x, y)$ of a trajectory of the dynamical system (6.12) with the potential given by (7.2) with $\omega^{2}=1.06$. The particle starts at the point marked by the black square, $\left(x_{0}, y_{0}\right)=(1,0)$, with an initial velocity $\left(u_{0}, v_{0}\right)=(0,1)$. The very slightly eccentric ellipse surrounding the orbit is the region $\Phi \leqslant E_{0} \equiv\left(\left(u_{0}^{2}+v_{0}^{2}\right)+\Phi\left(t_{0}\right)\right) / 2$, to which the particle has sufficient energy to go. In fact, due to the decoupling of (7.3), energy is conserved separately for the motion in $x$ and $y$, so the orbit is contained within the rectangle $\mathscr{D}$. Here $\omega$ is irrational, so the orbit will eventually cover $\mathscr{D}$ densely. With self-intersections this orbit clearly cannot be a streamline of a stationary flow.

The existence of solutions is an issue even for the nearly planar case studied in $\S 6.2$. As a prelude to considering boundary value problems involving (6.11), let us make a few remarks on the boundary value problem for 'ordinary' stationary inviscid incompressible flows. Consider the Euler equation and the continuity equation in a compact domain in the plane with boundary conditions giving the normal flow velocity through the boundary. Assuming irrotational flow, the problem reduces to solving the Laplace equation on the domain with Neumann boundary conditions, which is possible under rather general conditions, for example the total influx to the domain must be zero. For a mathematical discussion, see Courant \& Hilbert (1989, vol. II ch. IV). While this irrotational flow may not be the only solution to the flow problem, we know at least that a solution exists.

Boundary value problems for (6.11) are somewhat different. We now give an example of a boundary value problem which apparently has no solution at all. Consider (6.11) on the half-plane $x>-1$ with the boundary condition $u=0$ at $x=-1$, see figure $2(a)$. We take the potential to describe a circular harmonic well centred at the origin,

$$
\boldsymbol{f}=0, \quad \Phi=\frac{1}{2}\left(x^{2}+y^{2}\right)
$$


Note that while we now consider general (asymmetric) solutions and are working in the limit of a nearly planar surface, this potential is analogous to those considered in $\S 5$. Each component of (6.12) is simply an unforced harmonic oscillator of unit angular velocity, i.e $\ddot{x}_{i}+x_{i}=0$. So any solution to (6.12) describes a particle moving on an ellipse centred at the origin. As shown in figure 2, the trajectories of any two particles starting at distinct points on the boundary will intersect and have different velocities at the points of intersection, in conflict with the condition that the velocity depends on the position alone. So it appears that this boundary value problem has no solutions at all. Note that other choices of boundaries of prescribed normal velocities may yield a well-posed boundary value problem. For example, applying no-penetration boundary conditions to one of the elliptic trajectories from (6.12), we can find a solution, with streamlines consisting of smaller versions of the boundary curve.

A further complication becomes apparent if we break the symmetry of (7.1) slightly and take

$$
\boldsymbol{f}=0, \quad \Phi=\frac{1}{2}\left(x^{2}+\omega^{2} y^{2}\right),
$$

where $x=x_{1}$ and $y=x_{2}$, and $\omega=1+\delta, \delta \ll 1$. With (7.2), (6.12) separates into two harmonic oscillators

$$
\begin{aligned}
& \ddot{x}=-x, \\
& \ddot{y}=-\omega^{2} y,
\end{aligned}
$$

and the exact solution is straightforward. With the initial condition $x(0)=x_{0}, y(0)=y_{0}$, $\dot{x}(0)=u_{0}$ and $\dot{y}(0)=v_{0}$ in terms of real numbers $x_{0}, y_{0}, u_{0}, v_{0}$, the solution is

$$
\begin{aligned}
& x(t)=\left(x_{0}+\mathrm{i} u_{0}\right) \mathrm{e}^{-\mathrm{i} t}, \\
& y(t)=\left(y_{0}+\mathrm{i} \frac{v_{0}}{\omega}\right) \mathrm{e}^{-\mathrm{i} \omega t},
\end{aligned}
$$

where the real part of (7.4) is understood as the position. The motion is clearly bounded to a rectangle $\mathscr{D}=\left\{(x, y):|x| \leqslant x_{M} \wedge|y| \leqslant y_{M}\right\}$ with $x_{M}=\sqrt{x_{0}^{2}+u_{0}^{2}}$ and $y_{M}=\sqrt{y_{0}^{2}+v_{0}^{2} / \omega^{2}}$. If $\omega$ is rational and expressed as an irreducible fraction $p / q$, the motion (7.4) will be periodic with period $2 \pi q$. During one such period, $x$ will oscillate $q$ times while $y$ will oscillate $p$ times. In the process, the orbit will necessarily self-intersect several times, if $q$ is large. In the case when $\omega$ is irrational, the system will never return to the initial condition, and the motion is quasi-periodic. The orbit will then eventually cover $\mathscr{D}$ densely.

A set of orbits from (7.3) is shown in figure 2. As the trajectory meanders around in the rectangle $\mathscr{D}$, it undergoes several self-intersections. Such an orbit is clearly not a permissible streamline of a time-independent flow. Introducing non-harmonic terms in $\Phi$ in (7.2) only makes the situation worse, since we shall find chaotic orbits with self-intersections irregularly distributed, and thus it becomes even harder to find a domain where the streamlines do not intersect.

Apparently, the linearized version of (4.12) with (7.2) has no solution with anything even resembling closed streamlines. On the other hand, given a potential and a domain with a given boundary curve, we can construct a well-posed boundary value problem, if we choose the right boundary condition. For if we inject a particle at the boundary with a sufficiently large initial speed $\boldsymbol{v}_{0}$, the potential gradient forcing becomes a small perturbation, and the trajectories will approach straight lines in the case $f=0$ and segments of circles with radius $\left\|\boldsymbol{v}_{0}\right\| /\|\boldsymbol{f}\|$ otherwise. Clearly, we can always foliate the domain with trajectories of this form. So, while some boundary value problems cannot 
be solved, any particular bounded region does have an associated well-posed boundary value problem. Finally, we note that the nonlinear equations (4.12) will presumably retain many of the above properties as long as the surfaces are not too violently curved. This is ensured by the KAM theorem (see e.g. Ott 1993) which implies that invariant tori can persist even in non-integrable systems.

\section{Conclusion}

We have shown that it is possible to write down a self-contained set of equations for the flow on a stationary free surface of an ideal fluid. It is given by (4.12), which is the main result of the paper. We have shown how to include surface tension and velocity-dependent forces such as the Coriolis force, and demonstrated that the formalism provides a practical tool for determining actual flows on surfaces of simple shapes. We also showed that it can be used to predict that given surface shapes, boundary conditions or domains preclude the existence of a single-valued surface velocity field. Here the analogy to Hamiltonian particle dynamics with two degrees of freedom is very useful. The latter problem can always be solved with given initial conditions, and hence the question of existence of a velocity field on the surface becomes a question of projecting from the energy surface of the four-dimensional phase space of the dynamical system to the two-dimensional space of the surface, without intersections. In the present paper, we have given a preliminary analysis of this problem, and we believe that there are ample possibilities for developing this approach further in future work.

Finally, we mention that in modelling sea currents one is faced with a problem very similar to ours. Here satellite measurements of the free-surface elevation (altimetry) on a grid of points around the globe is used to predict the ocean currents. It is customary (see e.g. Ray 2001) to work in the linear, geostrophic approximation, where the inertial term (the left-hand side of (6.11)) is ignored, and the Coriolis term balances the forcing on the surface flow. If one were to include the nonlinear terms, one might in principle find that the surface shapes assumed cannot actually support the predicted flows! In the present state of our theory, however, we cannot perform such an analysis since we would first have to build in effects like time dependence and wind stresses, which are important for the ocean currents.

\section{Appendix. The form of the surface flow equations in simple situations}

\section{A.1. Cartesian base coordinates}

We shall now give an example of the general equations, (4.12), for the surface flow derived in the main body of the paper. Consider a free surface parameterized by $\left(x^{1}, x^{2}\right) \equiv(x, y)$ so the Cartesian laboratory coordinates $y^{\alpha}$ are

$$
\left(y^{1}, y^{2}, y^{3}\right)=(x, y, h(x, y)) .
$$

The two-dimensional metric tensor and its determinant for the surface manifold are then, by (4.6),

$$
\begin{aligned}
g_{i j} & =\left(\begin{array}{cc}
1+h_{x}^{2} & h_{x} h_{y} \\
h_{x} h_{y} & 1+h_{y}^{2}
\end{array}\right), \\
g \equiv|g| & =1+h_{x}^{2}+h_{y}^{2},
\end{aligned}
$$


where subscript $i$ denotes differentiation with respect to $x^{i}$. The $y^{\alpha}$ coordinate expression of the dual basis vectors (recall that the velocity vector is $V^{i} \boldsymbol{e}_{i}$ ) is $\boldsymbol{e}_{x}=\left(1,0, h_{x}\right), \boldsymbol{e}_{y}=\left(0,1, h_{y}\right)$. We denote the velocity vector components by $\left(V^{1}, V^{2}\right) \equiv(U, V)$.

The coordinate system is assumed to rotate in the counterclockwise direction about the $y^{3}$-axis with a constant angular velocity $\Omega$. In order to compute the Coriolis force term, we shall need $\Omega^{3}$ in terms of the surface-adapted coordinates defined at the beginning of $\S 4$, where $\boldsymbol{e}_{3}=1 / \sqrt{g} \cdot\left(-h_{x},-h_{y}, 1\right)$ in $y^{\alpha}$-coordinates. Thus, $\Omega^{3}=\Omega \boldsymbol{e}_{3} \cdot(0,0,1)=\Omega / \sqrt{g}$. Now, (4.12) becomes, for the $x$ - and $y$-directions, respectively:

$$
\begin{aligned}
\left(U U_{x}+V U_{y}\right)+h_{x} M= & \frac{1}{g}\left(-\left(1+h_{y}^{2}\right) \Phi_{x}+h_{x} h_{y} \Phi_{y}\right) \\
& +2 \Omega \frac{1}{g}\left(h_{x} h_{y} U+\left(1+h_{y}^{2}\right) V\right), \\
\left(U V_{x}+V V_{y}\right)+h_{y} M= & \frac{1}{g}\left(h_{x} h_{y} \Phi_{x}-\left(1+h_{x}^{2}\right) \Phi_{y}\right) \\
& -2 \Omega \frac{1}{g}\left(\left(1+h_{x}^{2}\right) U+h_{x} h_{y} V\right),
\end{aligned}
$$

where

$$
M=\frac{1}{g}\left(h_{x x} U^{2}+2 h_{x y} U V+h_{y y} V^{2}\right) .
$$

If we include a centrifugal force, a gravitational potential $g_{0} h(x, y)$ and a surface tension $\gamma$ with the associated Young-Laplace pressure on the free surface, and denote the constant liquid mass density by $\rho_{0}$, we have

$$
\Phi(x, y)=-\frac{1}{2} \Omega^{2}\left(x^{2}+y^{2}\right)+g_{0} h(x, y)-2{\frac{\gamma}{\rho_{0}}}_{0} H(x, y),
$$

where $H$ is the mean curvature (positive in the $y^{3}$-direction) of the free surface and must be computed from (A 1), for example using (4.8).

\section{A.2. Polar base coordinates}

We do the same for polar $(\rho, \theta)$ base coordinates, i.e.

$$
\left(y^{1}, y^{2}, y^{3}\right)=(\rho \cos \theta, \rho \sin \theta, h(\rho, \theta)),
$$

with the metric

$$
\begin{aligned}
g_{i j} & =\left(\begin{array}{cc}
1+h_{\rho}^{2} & h_{\rho} h_{\theta} \\
h_{\rho} h_{\theta} & \rho^{2}+h_{\theta}^{2}
\end{array}\right), \\
g & =\rho^{2}+\rho^{2} h_{\rho}^{2}+h_{\theta}^{2} .
\end{aligned}
$$

The dual basis expressed in $y^{\alpha}$-coordinates is $\boldsymbol{e}_{\rho}=\left(\cos \theta, \sin \theta, h_{\rho}\right), \quad \boldsymbol{e}_{\theta}=$ $\left(-\rho \sin \theta, \rho \cos \theta, h_{\theta}\right)$. The relevant component of $\Omega^{\mu}$, corresponding to a rotation vector of magnitude $\Omega$ in the positive $y^{3}$-direction, is $\Omega^{3}=\rho \Omega / \sqrt{g}$. Now, (4.12) 
becomes, for $\rho$ and $\theta$, respectively:

$$
\begin{aligned}
& \left(U U_{\rho}+V U_{\theta}\right)+\frac{1}{g}\left[\rho^{2} h_{\rho} h_{\rho \rho} U^{2}+2 \rho h_{\rho}\left(\rho h_{\rho \theta}-h_{\theta}\right) U V+\rho\left(\rho h_{\rho} h_{\theta \theta}-\rho^{2}-h_{\theta}^{2}\right) V^{2}\right] \\
& =\frac{1}{g}\left[-\left(h_{\theta}^{2}+\rho^{2}\right) \Phi_{\rho}+h_{\rho} h_{\theta} \Phi_{\theta}\right]+2 \Omega \frac{\rho}{g}\left[h_{\rho} h_{\theta} U+\left(\rho^{2}+h_{\theta}^{2}\right) V\right], \\
& \left(U V_{\rho}+V V_{\theta}\right)+\frac{1}{g}\left[h_{\theta} h_{\rho \rho} U^{2}+2\left(\rho\left(1+h_{\rho}^{2}\right)+h_{\theta} h_{\rho \theta}\right) U V+h_{\theta}\left(\rho h_{\rho}+h_{\theta \theta}\right) V^{2}\right] \\
& =\frac{1}{g}\left[h_{\rho} h_{\theta} \Phi_{\rho}-\left(1+h_{\rho}^{2}\right) \Phi_{\theta}\right]-2 \Omega \frac{\rho}{g}\left[\left(1+h_{\rho}^{2}\right) U+h_{\rho} h_{\theta} V\right] .
\end{aligned}
$$

Including the same physical effects as in the previous section, cf. (A 6), we have the potential

$$
\Phi(\rho, \theta)=-\frac{1}{2} \Omega^{2} \rho^{2}+g_{0} h(\rho, \theta)-2 \frac{\gamma}{\rho_{0}} H(\rho, \theta)
$$

\section{REFERENCES}

ARIS, R. 1962 Vectors, Tensors and the Basic Equations of Fluid Mechanics. Prentice Hall.

ARnold, V. I. 1978 Mathematical Methods of Classical Mechanics. Springer.

Bergmann, R., Tophøj, L., Homan, T. A. M., Hersen, P., Andersen, A. \& Bohr, T. 2011 Polygon formation and surface flow on a rotating fluid surface. J. Fluid Mech. 679, 415-431.

do Carmo, M. P. 1976 Differential Geometry of Curves and Surfaces. Prentice-Hall.

Carroll, S. 2003 Spacetime and Geometry: An Introduction to General Relativity. Pearson.

Courant, R. \& Hilbert, D. 1989 Methods of Mathematical Physics. Wiley.

ILIN, A. A. 1991 The Navier-Stokes and Euler equations of two-dimensional closed manifolds. Math. USSR Sb. 69, 559-579.

Lebedev, L. P., Cloud, M. J. \& Eremeyev, V. A. 2010 Tensor Analysis with Applications in Mechanics. World Scientific.

Oтт, E. 1993 Chaos in Dynamical Systems. Cambridge University Press.

RAY, R. D. 2001 Inversion of oceanic tidal currents from measured elevations. J. Mar. Syst. 28 (1-2), 1-18.

Stoker, J. J. 1969 Differential Geometry. Wiley. 